\title{
Real-time decision-making in chronic illness branching simulation
}

\author{
Natalya Pasklinsky ${ }^{1}$, Ashley Graham-Perel ${ }^{1}$, Princess Villacarlos-Philip ${ }^{1}$, Maryann Slaka-Vella ${ }^{1}$, \\ Charles P. Tilley ${ }^{1,2}$ \\ ${ }^{1}$ Department of Clinical Simulation Learning, ${ }^{2}$ Calvary Hospital and Hospice, New York University Rory Meyers College of Nursing, New York, \\ NY, USA \\ Correspondence to: Natalya Pasklinsky. New York University Rory Meyers College of Nursing, 433 First Avenue, New York, NY 10010, USA. \\ Email: veksln01@nyu.edu.
}

\begin{abstract}
The United States (US) is facing a rapidly aging population that suffers from multiple chronic illnesses. To prepare nursing students to care for this increasingly complex, aging population nurse educators must develop curricula that incorporate both current technology and cutting-edge teaching pedagogies that facilitate development of real-time decision-making skills. Branching scenarios are simulations that mimic real-life; rapidly changing patient conditions unfold based on actual student decision-making. This challenges learners to adapt the nursing process based on subjective and objective assessments and utilize current technology to analyze multiple sources of patient data. As nursing students make decisions and act, the scenario branches, presenting them with immediate feedback on the outcomes. Branching simulation scenario designs incorporate multiple different mobile technologies with decision-support software that nursing students may access, such as real-time decision-support algorithms, evidence-based guidelines, telehealth, medication information resources, and electronic medical records (EMRs). Faculty at NYU Rory Meyers College of Nursing (NYU) have successfully incorporated mobile technology with decision-support software into branching simulations as a strategy to develop real-time clinical decision-making in the care of older adults with multiple chronic illnesses.
\end{abstract}

Keywords: Simulation learning; branching scenarios; real-time decision-making

Received: 21 October 2019; Accepted: 03 June 2020; Published: 20 January 2021.

doi: $10.21037 /$ mhealth-19-215

View this article at: http://dx.doi.org/10.21037/mhealth-19-215

\section{Introduction}

As the aging US population approaches senescence, the number of people 65 years or older that suffer from multiple chronic illnesses is rapidly rising $(1,2)$. By $2019,88 \%$ of people aged 65 years or older will have one or more chronic illnesses (2). Comprehensive nursing care of this population may maintain or improve quality of life by slowing chronic disease progression, prevent unnecessary hospitalizations, and avoid medication errors and treatment complications (3). Comprehensive nursing care of the older adult includes meticulous patient assessment, comprehensive care planning, interprofessional collaboration and communication, and patient and family-centered education. Importantly, comprehensive nursing care requires real-time provision of information to nursing staff to support evidence-based decision-making (4). Thus, access to, and utilization of healthcare technology is essential to teach future nursing professionals real-time decision-making, which requires filtering and analyzing multiple sources of patient data (vital signs, lab values, imaging, medications, and referrals), as well as reviewing evidence-based guidelines and policies and procedures. Therefore, in order to prepare nursing students to care for an increasingly complex, aging population, nurse educators must develop curricula that incorporates both current technology and cutting-edge teaching pedagogies in the development of learners' real-time decision-making skills.

Innovative advances in simulation techniques in baccalaureate nursing education have paved the way to support real-time-decision-making skill development in 
novice nurses, providing them with the tools to care for seriously ill older adults. Simulation-based teaching utilizes real-time decision making to develop nursing students' critical thinking and clinical reasoning skills in a safe and controlled environment (4,5). Branching simulation designs are effective pedagogies to teach real-time decision making in the care of older adults with a variety of chronic illnesses. Branching scenarios unfold based on student decision making, challenging learners to adapt the nursing process to rapidly changing patient conditions and data (6). Branching simulation scenario designs may incorporate the use of multiple different technologies that nursing students can choose to access, such as real-time decisionsupport algorithms, evidence-based guidelines, telehealth, medication information resources, and EMRs that supports clinical decision-making and comprehensive care plan development (6).

Faculty in undergraduate community health and acute care courses at NYU have successfully incorporated mobile technology into branching simulation scenarios by utilizing iPads and iPhones loaded with decision support software; Lippincott's DocuCare ${ }^{\odot}$, a simulated EMR that also provides access to a medication information database (Lippincott's Nursing Advisor ${ }^{\odot}$ ), and UpToDate ${ }^{\odot}$, an evidence-based electronic clinical resource tool that also has a medication information database (Lexicomp $\left.{ }^{\circledR}\right)$. This article will discuss the use of branching simulation scenario designs with mobile decision support technology to foster real-time decision making in undergraduate nursing curricula in the care of older adults with multiple chronic illnesses.

\section{Integrating mobile technology into branching simulations}

Branching simulation is a highly interactive form of learning (6). It challenges the students, requires them to make a real-time decision, and then immediately presents to them the patient outcomes. Each outcome produces new challenges and more choices. As the learner makes decisions and intervenes in a scenario, the case unfolds in unpredictable ways, thereby making such learning interaction engaging (6). A well-designed branching scenario involves real-time decision making involving mobile decision-support technology with immediate feedback to students on the patient outcomes resulting from their decisions and subsequent interventions (6).

In "real life" emergent cases, Registered Nurses (RNs) face a myriad of subjective and objective data that influences their decision making and the nursing interventions they choose. Clinical decision support (CDS) tools are designed to help process enormous amounts of digital data to suggest next steps for nursing interventions, alert nurses to available information they may not have noted, or catch potential problems, such as dangerous medication interactions (7). CDS is any tool that provides clinicians, patients, caregivers, or other members of the healthcare team with information that is filtered or targeted to a specific person or situation (7). NYU simulation faculty at the Clinical Simulation Learning Center (CSLC) integrate iPad technology (Lippincott's DocuCare ${ }^{\odot}$ EMR and Nursing Advisor $\left.{ }^{\odot}\right)$ and smart phone decision support software $\left(\mathrm{UpToDate}^{\odot}{ }\right.$ Lexicomp $\left.^{\odot}\right)$ to develop real-time decision making. Simulation scenario design incorporates CDS to improve care quality, avoid errors (medication, treatment) or adverse events, and allows nursing students to be more efficient $(7,8)$.

\section{Branching scenario development: community health}

The community health nursing simulation experience provides nursing students with the opportunity to apply their acquired assessment, critical thinking, and communication skills to the provision of geriatric care outside of the acute care setting. Simulation experiences are important, as nursing students may not have the opportunity to engage in independent decision making during clinical practicum experiences due to workplace demands, time constraints, and additional concerns about patient safety (9). The NYU simulation faculty follow the International Nursing Association for Clinical Simulation and Learning (INACSL) Outcomes and Objectives and Design Standards (Box 1) to develop all simulation objectives and scenarios (10). The undergraduate program uses a standardized template to develop all simulation scenarios.

The community telehealth scenario involves the care of a 75 -year-old client recently discharged to home following a 4-day hospitalization for decompensated heart failure (HF). This client has a history of uncontrolled diabetes mellitus (DM), hypertension (HTN), hyperlipidemia (HLD), as well as a non-healing diabetic heel ulcer. The learning objectives for this 90-minute scenario are outlined in Box 2. A presimulation case study assignment, focusing on the key concepts of the scenario is completed prior to simulation. Six to eight students are involved in the branching simulation experience, which includes the used of mobile technology to access patient data (Telehealth) and CDS 
(UpToDate ${ }^{\circledR}$, Lippincott’s Nursing Advisor $^{\circledR}$ ).

Box 1 INACSL standards of best practice: simulation design

1. Perform a needs assessment to provide foundational evidence

2. Construct measurable objectives

3. Structure the format based on the purpose, theory, and appropriate simulation modality

4. Design the scenario or case to provide the context for the simulation experience

5. Use the appropriate type of fidelity to create the required perception of realism

6. Maintain a facilitative approach that is participant-centered and driven by the objectives

7. Begin the simulation with a rebreeding

8. Follow the simulation with a debriefing session

9. Include an evaluation of the participants, experience, facility, and facilitators

10. Provide preparation materials and resources to meet objectives

11. Pilot test the simulation prior to full implementation

Box 2 Student learning objectives: community telehealth simulation

At the conclusion of this simulation session, the student will

1. Analyze the telehealth data and utilize clinical decision support technology to formulate a plan of care for a patient with multiple chronic illnesses

2. Utilize the patient's discharge instructions and Lippincott's Nursing Advisor ${ }^{\odot}$ to perform medication reconciliation and evaluate the medication regime for drug-drug interactions

3. Verify understanding of medication regimen and provide needed education.

4. Perform a dressing change to the diabetic heel ulcer and formulate a teaching plan: wound care

5. Provide education on signs on symptoms of heart failure, hyperglycemia, and telehealth monitoring of vital signs, weights, and blood sugars

The first student establishes the reason and plan for the visit, reviews the discharge orders, and performs a health history and physical assessment. The patient, a high-fidelity manikin, is programmed with abnormal assessment findings: presence of crackles and worsening edema. High-fidelity manikins allow for the real-time deterioration of a patient and provide students the opportunity to rapidly assess the situation, grasp the gravitas, and perform evidence-based interventions in a safe learning environment (5). Review of the telehealth data reveals an elevated blood pressure, heart rate and respiratory rate, elevated blood sugars and rapid weight gain. An example of branching would be acute decompensation of the patient in the home if the student misses or incorrectly interprets these key findings. The student may choose to access CDS technology, such as $\mathrm{UpToDate}^{\odot}$, to review the physiologic meaning of these findings or consult the HF guidelines for next steps in careplanning. As the scenario progresses, the students must identify medical errors during medication reconciliation and use Lippincott's Nursing Advisor ${ }^{\odot}$ to check for drugdrug interactions, side effects, and may choose to refer to the computerized patient education guides.

As the scenario progresses, students are expected to recognize the client's continued decline and provide appropriate interventions (calling the physician for medication clarification, report deterioration, etc.) which will result in improvement, however if they fail to do so, the patient will continue to decompensate, necessitating rehospitalization. The CDS technology empowers the nursing students to engage in critical, real-time decision-making, vital when escalating the treatment of a deteriorating patient (11).

During debriefing students receive real-time feedback on their communication and use of CSD to support evidence-based interventions, patient education, and comprehensive plans of care. During debriefing, students discuss the positive aspects of their performance as well as mistakes while feeling supported by faculty and peers. This discussion helps students to consider alternative realtime decisions, and understand the different ways CSD can support the decision making process.

\section{Branching scenario development: acute care}

The acute care nursing scenario revolves around a 75-yearold client with long-standing chronic obstructive pulmonary disease (COPD) and a tracheostomy who has become dyspneic and is brought to the Emergency Department (ED) by paramedics. The patient is admitted to the ED for initial treatment of an acute exacerbation of his COPD. The learning objectives for this 90 -minute scenario is outlined in 
Box 3. An unfolding case study assignment, focusing on the key concepts of the rapidly-deteriorating-patient scenario is completed prior to simulation. Six to eight students are involved in the branching simulation experience, which includes the used of mobile technology to access patient data (DocuCare ${ }^{\odot}$ EMR) and CDS (UpToDate ${ }^{\odot}$, Lippincott's Nursing Advisor ${ }^{\circledR}$ ).

Box 3 Student learning objectives: managing rapid deterioration of the COPD patient

At the conclusion of this simulation session, the student will

1. Conduct a focused neurologic and cardio-pulmonary assessment for a rapidly deteriorating patient with COPD exacerbation

2. Analyze the vital signs, EKG, and laboratory data then utilize clinical decision support technology (UpToDate ${ }^{\mathbb{Q}}$ ) to formulate a plan of care for a rapidly deteriorating, chronically ill older adult

3. Utilize the patient's DocuCare ${ }^{\odot}$ EMR and Lippincott's Nursing Advisor ${ }^{\odot}$ to perform medication reconciliation and evaluate the medication regime for drug-drug interactions

4. Verify the correct tracheostomy care procedure in DocuCare $^{\odot}$ and demonstrate tracheostomy care

5. Demonstrate effective communication strategies with a non-verbal patient that has a tracheostomy

State one begins with a patient in respiratory distress admitted to the Emergency Department who requires emergency intervention. Students must make real-time decisions in this fast-paced clinical environment using a constant stream of digital clinical data from multiple technological sources; the cardiac monitor, vital sign machine, and the DocuCare ${ }^{\odot}$ EMR. As the patient's medications and interventions are ordered, the students must use the CDS technology (UpToDate ${ }^{\odot}$, Lippincott's Nursing Advisor $^{\odot}$ ) to verify these interventions and medications are appropriate for a rapidly deteriorating, chronically ill older adult. For example, the initial order for the infusion of intravenous fluids is too extreme, a medical error that if the students fail to recognize, leads the scenario to branch into further deterioration of the patient's breathing status and possible cardio-pulmonary arrest. If the students intervene appropriately, the scenario evolves to state two where the patient shows signs of improvement in their medical condition. The clinical decision-making in the case of the rapidly deteriorating patient is challenging even for seasoned providers and especially vexing for students $(12,13)$. This branching simulation provides an excellent opportunity to train students to utilize mobile technology that supports rapid, real-time decision-making in emergency situations that will positively influence patient outcomes (14).

\section{Conclusions}

The aging population in the US presents unique challenges to nurse educators as they prepare future nurses to deliver comprehensive care to older adults with multiple chronic illnesses. Branching simulation designs that utilize CDS tools are effective and innovative strategies to help learners develop real-time decision making by teaching them to access, filter, and analyze large amounts of digital patient data. Integrating current mobile technology into nursing curricula helps bridge the gap between the classroom and the fast-paced, complex clinical environment. The integration of mobile technology loaded with CDS software into branching scenarios is also a powerful tool for educators to promote evidence-based practice. This creative simulation design can be used to teach nursing students how to immediately access data, guidelines, and policies needed to formulate safe, comprehensive plans of care for our aging, complex patient population.

\section{Acknowledgments}

Funding: This work was supported by the Hugoton Foundation, to integrate palliative care of the seriously ill into nursing simulation curriculum.

\section{Footnote}

Provenance and Peer Review: This article was commissioned by the Guest Editor (Mei R. Fu) for the series "Real-Time Detection and Management of Chronic Illnesses" published in mHealth. The article was sent for external peer review organized by the Guest Editor and the editorial office.

Conflicts of Interest: All authors have completed the ICMJE uniform disclosure form (available at http://dx.doi. org/10.21037/mhealth-19-215). The series "Real-Time Detection and Management of Chronic Illnesses" was commissioned by the editorial office without any funding or sponsorship. The authors have no other conflicts of interest 
to declare.

Ethical Statement: The authors are accountable for all aspects of the work in ensuring that questions related to the accuracy or integrity of any part of the work are appropriately investigated and resolved.

Open Access Statement: This is an Open Access article distributed in accordance with the Creative Commons Attribution-NonCommercial-NoDerivs 4.0 International License (CC BY-NC-ND 4.0), which permits the noncommercial replication and distribution of the article with the strict proviso that no changes or edits are made and the original work is properly cited (including links to both the formal publication through the relevant DOI and the license). See: https://creativecommons.org/licenses/by-nc-nd/4.0/.

\section{References}

1. Devitt M. CDC data show U.S. life expectancy continues to decline. Am Fam Physician 2018. Available online: https://www.aafp.org/news/health-of-the public/20181210lifeexpectdrop.html

2. Centers for Disease Control and Prevention. Data and statistics. 2019. Available online: https://www.cdc.gov/ chronicdisease/index.htm

3. Hughes RG. Nurses at the "sharp end" of patient care. Patient safety and quality: An evidence-based handbook for nurses. Agency for Healthcare Research and Quality (US); Rockville (MD), 2008. Available online: https://www. ncbi.nlm.nih.gov/books/NBK2672/

4. Hee-Ok P, Insook L. Enhancing critical thinking through simulation problem based learning in nursing education. Indian J Science \& Technology 2016;9:2-7.

doi: $10.21037 /$ mhealth-19-215

Cite this article as: Pasklinsky N, Graham-Perel A, VillacarlosPhilip P, Slaka-Vella M, Tilley CP. Real-time decision-making in chronic illness branching simulation. mHealth 2021;7:14.
5. Bliss M, Aitken LM. Does simulation enhance nurses' ability to assess deteriorating patients? Nurse Educ Pract 2018;28:20-6.

6. Kovach CR, Rababa M. Using branching simulations in treatment fidelity plans. Res Gerontol Nurs 2014;7:216-23.

7. Bresnick J. Understanding the basics of clinical decision support systems. Health IT. Available online: https:// healthitanalytics.com/features/understanding-the-basicsof-clinical-decision-support-systems

8. Schubert C. Effect of simulation on nursing knowledge and critical thinking in failure to rescue events. J Contin Educ Nurs 2012;43:467-71.

9. Kelly MA, Forber J, Conlon L, et al. Empowering the registered nurses of tomorrow: Students' perspectives of a simulation experience for recognizing and managing a deteriorating patient. Nurse Educ Today 2014;34:724-9.

10. INACSL Standards Committee. INACSL standards of best practice: Simulation, simulation design. Clin Simul Nurs 2016;12:S5-S12.

11. Liaw SY, Sherpbier A, Klainin-Yobas P, et al. A review of educational strategies to improve nurse's role in recognizing and responding to deteriorating patients. Int Nurs Rev 2011;58:296-303.

12. Genuino MJ. Effects of simulation-based educational program in improving the nurses' self-efficacy in caring for patients' with COPD and CHF in a post-acute care (PACU) setting. Appl Nurs Res 2016;39:53-7.

13. Woda A, Hansen J, Paquette M, et al. The impact of simulation sequencing on perceived clinical decision making. Nurse Educ Today 2017;26:33-8.

14. Young J. Effects of simulation on nursing students' knowledge, clinical reasoning, and self-confidence. Korean J Adult Nurs 2015;27:604-11. 